Article

\title{
Functional Characterization and Evolutionary Analysis of Glycine-Betaine Biosynthesis Pathway in Red Seaweed Pyropia yezoensis
}

\author{
Yunxiang Mao ${ }^{1,2,3,+}$, Nianci Chen ${ }^{1,3,+} \mathbb{C}$, Min Cao ${ }^{1,3}$, Rui Chen ${ }^{1,3}$, Xiaowei Guan ${ }^{1,3}$ \\ and Dongmei Wang $1,3, *$ \\ 1 Key Laboratory of Marine Genetics and Breeding (Ocean University of China) Ministry of Education, \\ Qingdao 266003, China; yxmao@ouc.edu.cn (Y.M.); 13969671852@163.com (N.C.); \\ caominjiayou@163.com (M.C.); cherryruirui99@163.com (R.C.); guanxw1995@163.com (X.G.) \\ 2 Laboratory for Marine Biology and Biotechnology, Qingdao National Laboratory for Marine Science and \\ Technology, Qingdao 266237, China \\ 3 College of Marine Life Sciences, Ocean University of China, Qingdao 266003, China \\ * Correspondence: wangdm@ouc.edu.cn; Tel.: +86-158-6429-1929 \\ + These authors contributed equally to this work.
}

Received: 26 December 2018; Accepted: 17 January 2019; Published: 21 January 2019

\begin{abstract}
The red seaweed Pyropia yezoensis is an ideal research model for dissecting the molecular mechanisms underlying its robust acclimation to abiotic stresses in intertidal zones. Glycine betaine (GB) was an important osmolyte in maintaining osmotic balance and stabilizing the quaternary structure of complex proteins under abiotic stresses (drought, salinity, etc.) in plants, animals, and bacteria. However, the existence and possible functions of GB in Pyropia remain elusive. In this study, we observed the rapid accumulation of GB in desiccated Pyropia blades, identifying its essential roles in protecting Pyropia cells against severe osmotic stress. Based on the available genomic and transcriptomic information of Pyropia, we computationally identified genes encoding the three key enzymes in the GB biosynthesis pathway: phosphoethanolamine $N$-methyltransferase (PEAMT), choline dehydrogenase (CDH), and betaine aldehyde dehydrogenase (BADH). Pyropia had an extraordinarily expanded gene copy number of $\mathrm{CDH}$ (up to seven) compared to other red algae. Phylogeny analysis revealed that in addition to the one conservative $\mathrm{CDH}$ in red algae, the other six might have originated from early gene duplication events. In dehydration stress, multiple CDH paralogs and PEAMT genes were coordinating up-regulated and shunted metabolic flux into GB biosynthesis. An elaborate molecular mechanism might be involved in the transcriptional regulation of these genes.
\end{abstract}

Keywords: Pyropia yezoensis; desiccation; betaine; PEAMT; CDH; BADH

\section{Introduction}

Plant performance and yield responses to water deficit stress conditions have been extensively studied [1-3]. Water deficiency causes cell metabolic disorder and cell membrane mechanical damage. A decline in leaf relative water content (RWC) reflects a loss of turgor that results in limited cell expansion and thereby reduced growth in crop plants [4,5]. Glycine betaine (GB) ( $\mathrm{N}, \mathrm{N}, \mathrm{N}$-trimethylglycine) is a quaternary ammonium compound found in bacteria, halophilic archaebacteria, marine invertebrates, plants, and mammals [6-9]. As an organic osmolyte, GB can maintain the osmotic balance and stabilize the quaternary structure of complex proteins under abiotic stresses. The protective role of GB has been widely reported in plants [10-12]. Treating plants with exogenous GB or increasing GB content in transgenic plants would greatly enhance their tolerance 
to osmotic stresses. Under chilling-stressed conditions, the heterologous expression of the betA gene significantly increased the tolerance of transgenic cotton seedlings to chilling injury through the accumulation of high levels of GB [13]. GB is also known to stabilize both photosystem II complexes and Rubisco under abiotic stress.

Four biosynthetic pathways for GB are known, three involve the oxidation of choline and one is the methylation of glycine [14,15]. In the choline oxidation pathway, GB is synthesized through a two-step oxidation of choline [16]. The first step converts choline into betaine aldehyde and is catalyzed by Rieske-type iron-sulphur enzyme choline monooxygenase (CMO; EC:1.14.15.7) in plants [17] and membrane-associated choline dehydrogenase (CDH; EC:1.1.99.1) in E.coli [18], animals, and Thalassiosira pseudonana [19]. The second step is catalyzed by nicotinamide adenine dinucleotide (NAD)-dependent betaine aldehyde dehydrogenase (BADH; EC:1.2.1.8) for the final biosynthesis of betaine. In soil bacterium Arthrobacter globiformis, betaine is directly catalyzed by choline oxidase (COD; EC:1.1.3.17) to degenerate hydrogen from choline. Besides this, three sequential methylation steps of phosphoethanolamine to generate phosphocholine, catalyzed by phosphoethanolamine $\mathrm{N}$-methyltransferase (PEAMT; EC:2.1.1.103), are also reported to be the rate limiting step in the choline-biosynthetic pathway [20,21]. The alternative GB biosynthetic pathway involves the three-step methylation of glycine, which is reported in halophilic microorganisms and also the diatom T. pseudonana $[22,23]$.

Pyropia yezoensis, previously known as Porphyra yezoensis [24], Rhodophyta, is a typical intertidal macroalgae. During daily low tides, P. yezoensis is routinely exposed to high levels of light, dehydration, and extreme fluctuations in temperature and osmotic pressure due to the seawater-to-air transition [25]. The blades can tolerate dehydration with a water loss of up to $85 \%$, and recover physiological activities immediately upon rehydration [26]. These characteristics make P. yezoensis an ideal model for studying the molecular mechanisms of stress tolerance in intertidal-zone seaweeds. However, to date, there have been few reports investigating the cellular osmolytes in P. yezoensis. Is GB present in Pyropia cells? What are the roles of the GB in response to osmotic stress in Pyropia? What influences the diversity and conservation of the GB biosynthesis pathway in Pyropia? To address these questions, in this study, we computationally identified genes involved in the betaine biosynthesis pathway. We identified the variation of enzymatic activities and transcriptions for key genes during dehydration as well as re-hydration processes.

\section{Results}

\subsection{GB Contents in Dehydrated P. yezoensis Blades}

To determine whether GB exists in red seaweed P. yezoensis and whether it is involved in desiccation tolerance, we detected the amounts of GB in the dehydration process. In 40-day old P. yezoensis blades, $6.720 \mu \mathrm{mol} / \mathrm{g}$ (fresh weight, FW) of GB was detected. The amount increased sharply to $19.924 \mu \mathrm{mol} / \mathrm{g}$ FW (a 2.96-fold increase compared to the control) when $50 \%$ of water was lost in the blades (Figure 1). However, after an extension of dehydration with water loss at $80 \%$, the GB amount dropped slightly, although there was still a 1.990-fold increase compared to the basal level. 


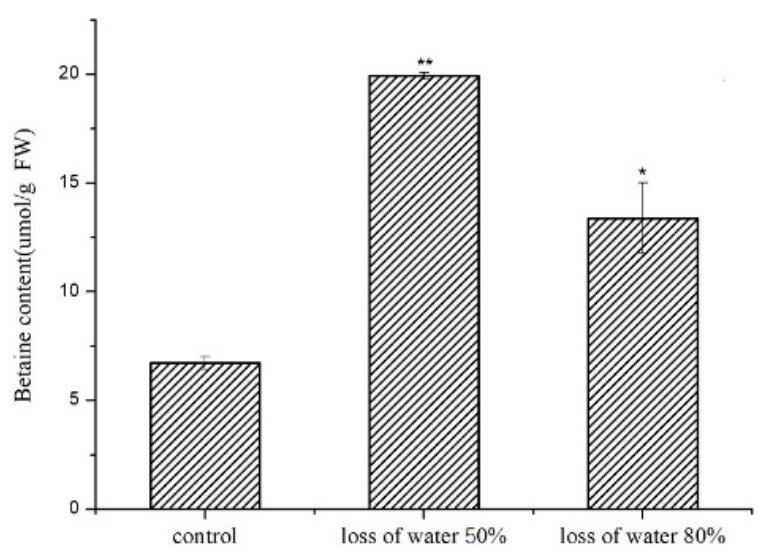

Figure 1. Profiles of glycine betaine (GB) contents in P. yezoensis blades under de-hydration stress. The seaweeds were desiccated until the total water content reached $50 \%$ and $80 \%$. Three replicates were produced for each treatment. Error bars indicate standard errors. The statistical significance compared to the control sample is indicated with one or two asterisks ( $p$ value is lower than $0.05\left(^{*}\right)$ or $\left.0.01{ }^{* *}\right)$, respectively).

\subsection{GB Biosynthesis Pathway in P. yezoensis}

The biosynthesis pathway of GB exhibited great diversity in plants, animals, bacteria as well as unicellular diatoms. Mainly two metabolic pathways lead to the GB biosynthesis, namely the choline oxidation pathway and the glycine methylation pathway. To identify candidate genes encoding the key enzymes related to GB biosynthesis in P. yezoensis and to further refer to its evolutionary history, we searched genomic and transcriptomic information published for P. yezoensis and other algal genomes including primary endosymbionts green algae and red algae, as well as the secondary endosymbionts diatom and Ectocarpus, via a blast program using the corresponding homologous genes from the above organisms as queries.

In the choline oxidation pathway, the first enzyme that catalyzes the oxidation of choline into choline aldehyde is choline dehydrogenase $(\mathrm{CDH})$. In P. yezoensis, we found seven genes encoding proteins with similarity to $\mathrm{CDH}$ homologs, while only three and one were found in Chondrus and the unicellular red algae Cyanodioschyzon, respectively. All of them harbored a GMC-oxred N conserved domain at the $N$-terminus (Table 1 ). We carried out the phylogenetic analysis to probe the evolutionary forces driving the expansion of $\mathrm{CDH}$ genes. In the evolutionary tree, the algal $\mathrm{CDHs}$ are grouped together, while the bacteria and animal homologs are in two other clusters, suggesting an ancient separation in the evolution of algal CDHs. The PyCDH1 showed a phylogeny with strong affiliation with its red-algal homologs. The other six $P y C D H s$ formed a sister group with two homologs from Chondrus (Figure 2). The most plausible explanation for the extraordinary expansion of CDH genes was the explosive gene duplication events in the P. yezoensis genome.

Table 1. Choline dehydrogenase (CDH) genes in P. yezoensis.

\begin{tabular}{ccccc}
\hline Gene & GenBank Entry & ORF (bp) & Amino Acids & Conserved Domain \\
\hline PyCDH1 & MK294537 & 3092 & 679 & GMC-oxred_N \\
PyCDH2 & MK294538 & 1761 & 586 & GMC-oxred_N \\
PyCDH3 & MK294539 & 2289 & 762 & GMC-oxred_N \\
PyCDH4 & MK294540 & 1710 & 557 & GMC-oxred_N \\
PyCDH5 & MK294541 & 1955 & 569 & GMC-oxred_N \\
PyCDH6 & MK294542 & 2291 & 572 & GMC-oxred_N \\
PyCDH7 & MK294543 & 1620 & 539 & GMC-oxred_N \\
\hline
\end{tabular}




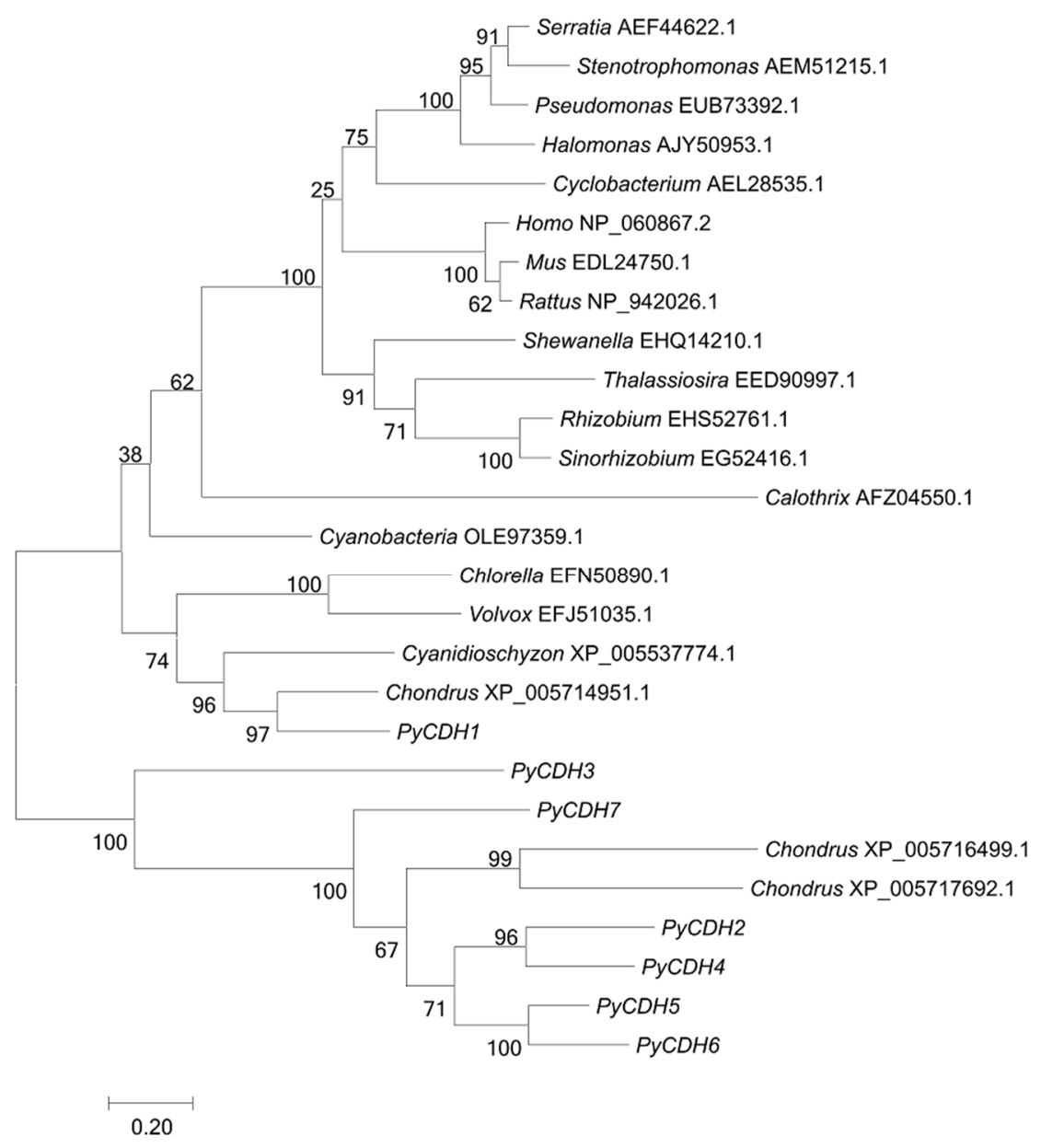

Figure 2. Phylogenetic analysis of $\mathrm{PyCDH}$ s and other choline dehydrogenase (CDHs). An unrooted phylogenetic tree was constructed using the maximum likelihood phylogenetic method in the MEGA version 7.0 software. The branch lengths are proportional to the evolutionary distances. The sequence of CDH genes include Cyanobacteria bacterium 13_1_20CM_4_61_6 (OLE97359.1), Thalassiosira pseudonana CCMP1335 (EED90997.1), Volvox carterif. nagariensis (EFJ51035.1), Calothrix sp. PCC 6303 (AFZ04550.1), Cyanidioschyzon merolae strain 10D (XP_005537774.1), Chlorella variabilis (EFN50890.1), Cyclobacterium marinum DSM 745 (AEL28535.1), Sinorhizobium meliloti AK83 (AEG52416.1), Stenotrophomonas maltophilia JV3 (AEM51215.1), Serratia plymuthica AS9 (AEF44622.1), Rhizobium sp. PDO1-076 (EHS52761.1), Halomonas sp. KO116 (AJY50953.1), Shewanella baltica OS183 (EHQ14210.1), Pseudomonas sp. GM41(2012) (EUB73392.1), Homo sapiens (NP_060867.2), Mus musculus (EDL24750.1), Rattus norvegicus (NP_942026.1), and Chondrus crispus (XP_005714951.1, XP_005717692.1, XP_005714951.1).

BADH catalyzes the last step converting betaine aldehyde into betaine. Thus, we also computationally identified the putative BADH genes in P. yezoensis. PyBADH contains an ORF of $2428 \mathrm{bp}$, encoding 691 amino acids. PyBADH protein harbors a conservative motif $\mathrm{x}-\mathrm{x}-\mathrm{x}-\mathrm{E}-\mathrm{L}-\mathrm{G}-\mathrm{G}-\mathrm{K}-\mathrm{x}-\mathrm{x}$ (Figure 3), which is characterized as dehydrogenation, and several residues that are characterized as being involved in $\mathrm{NAD}^{+}$binding and catalytic sites [27]. The phylogenetic analysis of BADHs showed that the $P y B A D H$ formed a close clade with unicellular red algae homologs (Figure 4).

PEAMT catalyzes the methylation of phosphoethanolamine to form choline, and is characterized as being essential for betaine biosynthesis in plants. In P. yezoensis, we found one putative PEAMT gene encoding 468 amino acids. The functional domain analysis of PyPEAMT showed that PEAMT belongs to the $\mathrm{N}$-methyltransferase superfamily. 

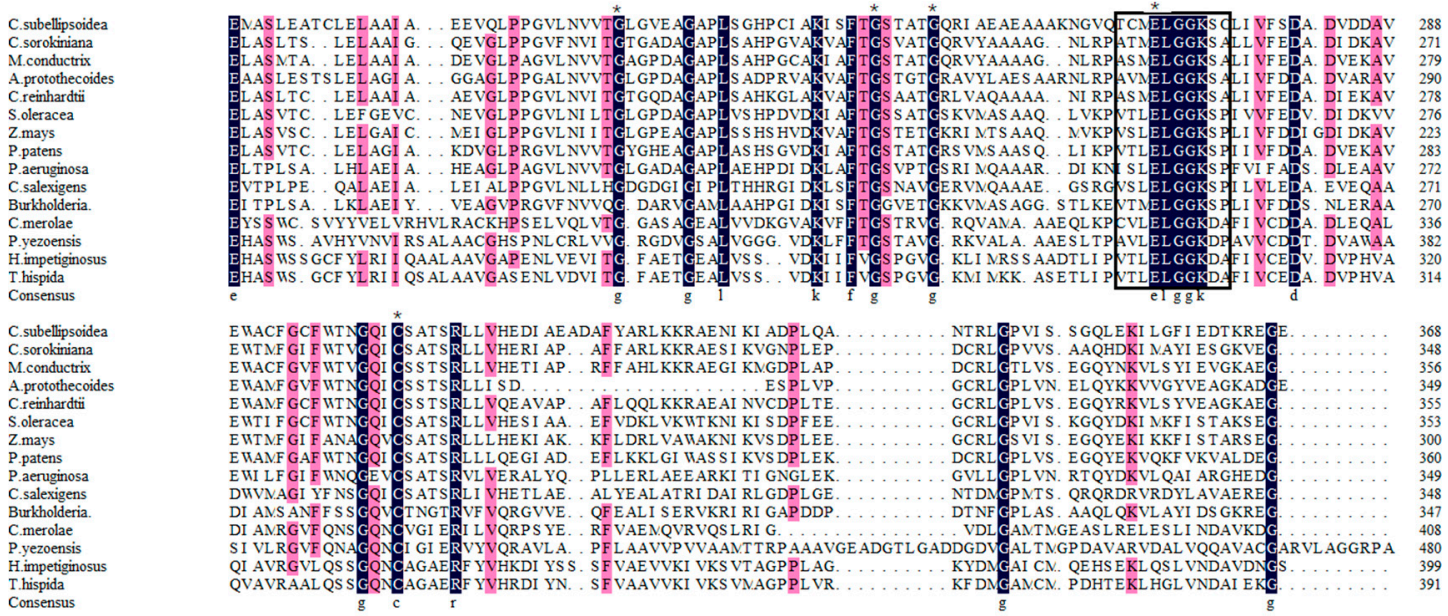

Figure 3. Multiple sequence alignment of betaine aldehyde dehydrogenase (BADH) amino acid sequences by using Clustal $X$ 2.0. The same amino acid residue is indicated in black font. Asterisks indicate putative residues involved in nicotinamide adenine dinucleotide $\left(\mathrm{NAD}^{+}\right)$binding, and boxes indicate highly conserved regions in the catalytic sites of various dehydrogenases. The sequence of BADH genes includes: Coccomyxa subellipsoidea C-169(XP005645499.1), Chlorella sorokiniana (PRW59377.1), Micractinium conductrix (PSC76875.1), Auxenochlorella protothecoides (XP011398091.1), Chlamydomonas reinhardtii (XP001699134.1), Spinacia oleracea (AAB41696.1), Zea mays (NP001157804.1), Physcomitrella patens (XP001756623.1), Chromohalobacter salexigens (YP574889.1), Burkholderia sp. MR(KIG10012.1), Cyanidioschyzon merolae strain 10D(XP005536893.1), Porphyra yezoensis, Handroanthus impetiginosus (PIN07780.1), Pseudomonas aeruginosa (ACD38844.1), and Tamarix hispida -2(AIL24124.1).

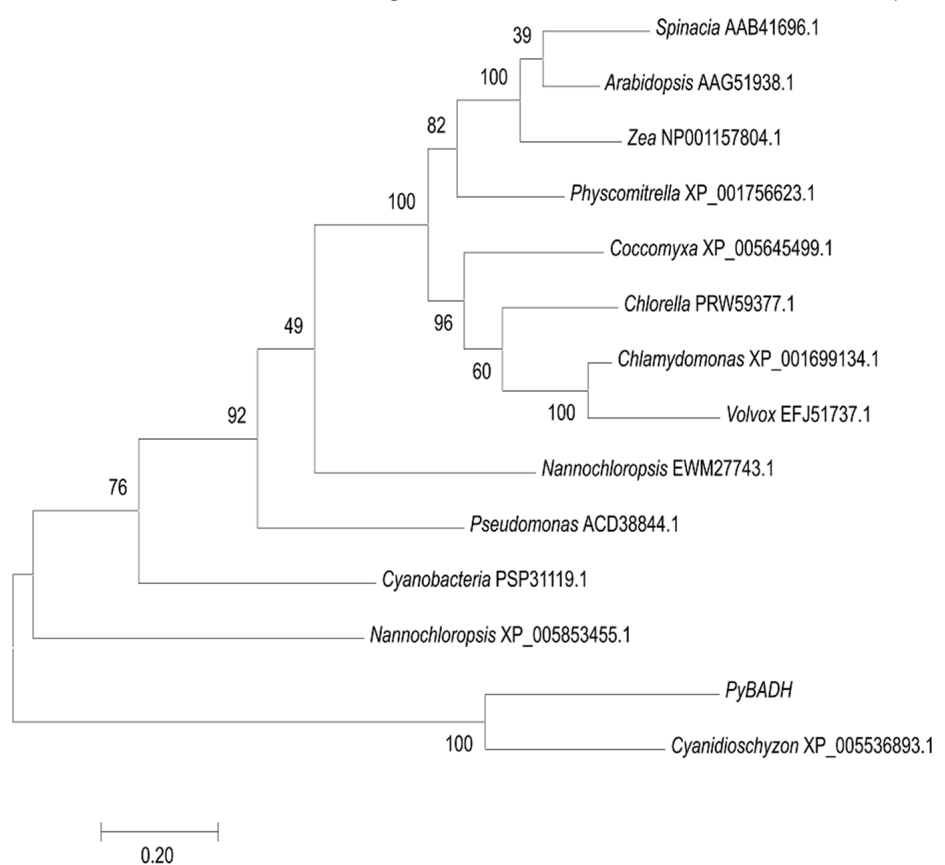

Figure 4. Phylogenetic analysis of $P y B A D H$ and other betaine aldehyde dehydrogenase (BADHs). An unrooted phylogenetic tree was constructed using the maximum likelihood phylogenetic method in the MEGA version 7.0 software. The branch lengths are proportional to the evolutionary distances. The sequence of BADH genes includes Spinacia oleracea (AAB41696.1), Pseudomonas aeruginosa (ACD38844.1), Zea mays (NP001157804.1), Physcomitrella patens (XP001756623.1), Chlamydomonas reinhardtii (XP001699134.1), Coccomyxa subellipsoidea C-169 (XP005645499.1), Chlorella sorokiniana (PRW59377.1), Cyanidioschyzon merolae strain 10D (XP005536893.1), Cyanobacteria bacterium SW_4_48_29 (PSP31119.1), Nannochloropsis gaditana (XP_005853455.1,EWM27743.1), Thalassiosira pseudonana (ACI64514.1), Volvox carteri f. nagariensis (EFJ51737.1), and Arabidopsis thaliana (AAG51938.1). 
In the glycine methylation pathway for GB biosynthesis, the sequential methylation is catalyzed by two AdoMet dependent methyltransferases in bacteria: glycine sarcosine methyltransferase (GSMT) and sarcosine dimethyl-glycine methyltransferase (SDMT). When blasting against $P$. yezoensis unigenes using bacteria homologs as the query, the best hit goes to PyPEAMT, as all of them have the conserved methyltransferase domain. However, in the phylogeny tree with both SDMT/DMT and PEAMT homologs included, the PYPEAMT was grouped in the PEAMT clade (Figure 5). Therefore, we deduced that the glycine methylation pathway might not exist in the P. yezoensis genome.

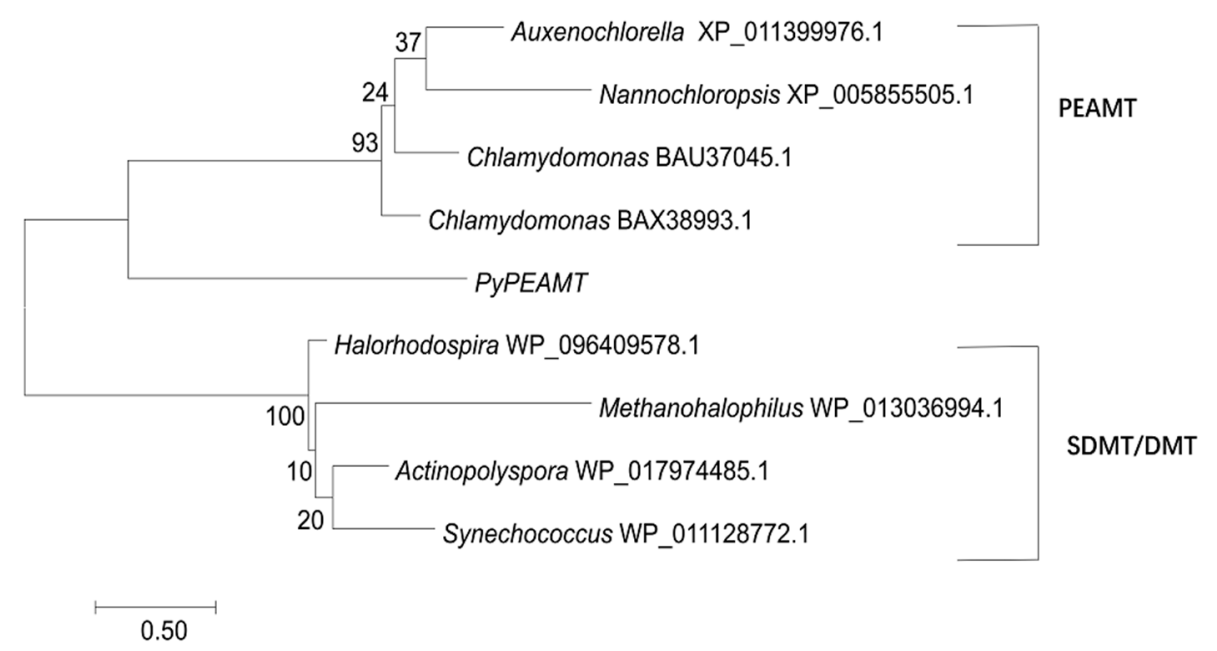

Figure 5. Phylogenetic analysis of PyPEAMT, other phosphoethanolamine N-methyltransferase (PEAMTs) and other glycine sarcosine methyltransferase (GSMT). An unrooted phylogenetic tree was constructed using the maximum likelihood phylogenetic method in the MEGA version 7.0 software. The branch lengths are proportional to the evolutionary distances. The sequence of relative genes includes Auxenochlorella protothecoides (XP_011399976.1), Nannochloropsis gaditana CCMP526 (XP_005855505.1), Chlamydomonas applanata (BAX38993.1), Chlamydomonas asymmetrica (BAU37045.1), Actinopolyspora halophila (WP_017974485.1), Halorhodospira halochloris (WP_096409578.1), Methanohalophilus mahii (WP_013036994.1), and Synechococcus sp. (WP_01128772.1).

\subsection{Transcriptional Variations of $C D H, B A D H$, and PEAMT Genes Under Dehydration}

We carried out real-time fluorescence quantitative polymerase chain reaction (PCR) to investigate the transcriptional variation of PEAMT, $\mathrm{BADH}$, and four $\mathrm{CDH}$ genes in P. yezoensis during the dehydration process. PEAMT exhibited a gradual up-regulation with the aggravation of water loss, and reached a peak at $80 \%$ water loss. A synchronized sharp increase in transcription at the onset of dehydration was observed in all the four $\mathrm{CDH}$ genes. Although they returned to the basal level thereafter, $\mathrm{CDH} 1, \mathrm{CDH} 2$, and $\mathrm{CDH} 3$ increased in the subsequent re-hydration, with $\mathrm{CDH} 3$ even rising to a 5.06-fold increase compared to the control. BADH only showed slight up-regulation at $30 \%$ water loss. Its transcription dropped thereafter and even went to half of the basal level in re-hydration (Figure 6a). 


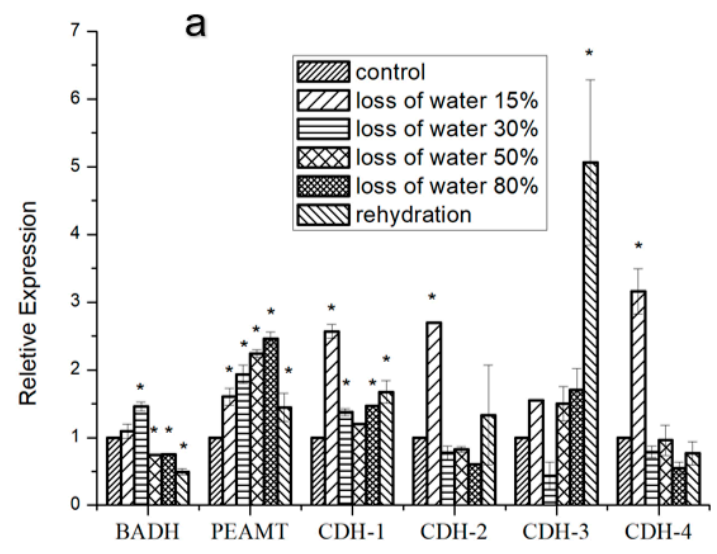

b

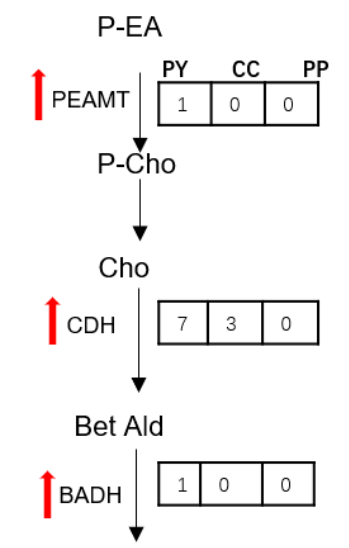

Glycine betaine(GB)

Figure 6. Profiles of putative GB biosynthetic genes. (a) Three replicates were produced for each treatment. The symbol ${ }^{*}$ represents significant differences from control values at $p<0.05$ (Student's t-test). (b) P-EA, phosphor-ethanolamine; P-Cho, phosphocholine; Cho, choline; Bet Ald, betaine-aldehyde; PY, Pyropia yezoensis; CC, Chondrus crispus; PP, Porphyridium purpureum. The red arrows indicate the transcriptional up-regulation under desiccation stress. The numbers in the squares refer to the gene copy numbers of each enzyme in Pyropia yezoensis, Chondrus crispus, and Porphyridium purpureum, respectively.

\subsection{CDH Activity under Dehydration Stress in P. yezoensis Seaweeds}

We further detected the enzymatic activity of $P$. yezoensis CDHs in desiccated blades and found that its variation was well correlated with the transcriptional levels. The $\mathrm{CDH}$ enzymatic activity increased immediately at the early stage of water loss (1.54-fold) and then slightly dropped to the basal level under severe dehydration. When rehydrated, the enzyme activity increased 1.4-fold again (Figure 7).

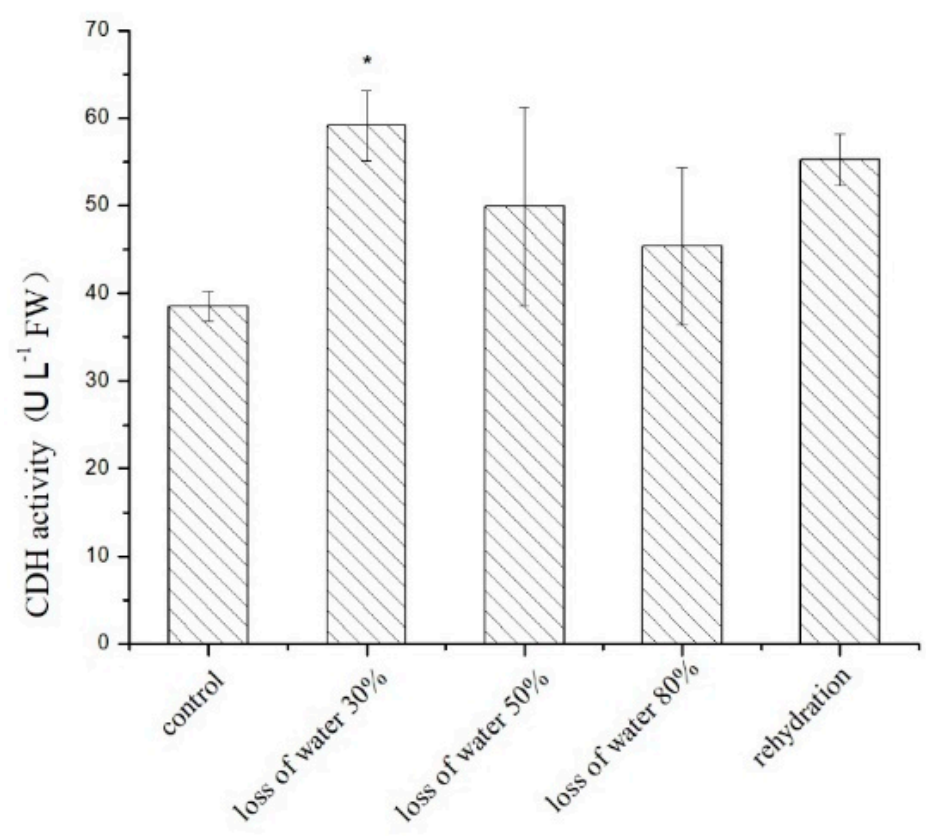

Figure 7. Profiles of $\mathrm{CDH}$ activity under dehydration stress in P. yezoensis. The algae reached water loss levels of $30 \pm 5 \%, 50 \pm 5 \%$, and $80 \pm 5 \%$, respectively, as well as rehydration after $80 \%$ water loss for $30 \mathrm{~min}$. Three replicates were produced for each treatment. The symbol * represents significant differences from control values at $p<0.05$ (Student's $t$-test). 


\section{Discussion}

The data presented in this study strongly indicates that glycine betaine, as a compatible solute, plays essential roles in protecting $P$. yezoensis against osmotic stress. This is the second algal species (the first red algae) reported to have a betaine biosynthesis pathway. However, in the diatom T. pseudonana, the enzymatic activity of $\mathrm{CDH}$ was not provided [19]. We also computationally identified homologous genes of PEAMT and BADH in other three red algae as well as green algae (Figure 6b). The wide occurrence of the choline oxidation pathway in organisms strongly suggests its evolutionary conservation. Conversely, the GB methylation pathway is present only in bacteria and the diatom T. pseudonana, as reported recently. The PEAMT gene in P. yezoensis has high protein similarity to bacteria SDMT and was doubted to be the putative SDMT homolog catalyzing the methylation of glycine. However, the phylogeny analysis did not support this argument. Moreover, at least two proteins (GSMT, SDMT /DMT) or one integrative peptide with two substrate-specific methyltransferase domains such as in T. pseudonana are required by the three-step methylation of glycine [19], while only one domain was found in PYPEAMT. Therefore, we deduced that the glycine methylation pathway is bacteria-specific and the diatom T. pseudonana might obtain this pathway occasionally via horizontal gene transfer, transposable elements, etc.

Choline, the precursor of betaine, could also go into either the lipid metabolic pathway or the acetylcholine biosynthesis. Choline dehydrogenase was the key enzyme to determine the metabolic direction of choline into betaine biosynthesis. In the P. yezoensis genome, up to seven $\mathrm{CDH}$ paralogs were identified, representing the highest gene dose among known animal, algal, and bacterial genomes. The increase in particular gene families was frequently reported in genomic analysis. Usually, several mechanisms have been proposed to play essential roles in obtaining new genetic materials for environmental adaptation, including genome-scale duplication, regional duplication due to homologous recombination, and illegitimate recombination, as well as horizontal gene transfer from bacteria or archaea. No evidence of genome duplication was observed in P. yezoensis. Besides, in the phylogeny analysis of $\mathrm{PyCDHs}$, they were clustered separately from bacteria $\mathrm{CDH}$ homologs, excluding the existence of horizontal gene transfer (HGT) events in the CDH expansion. Therefore, according to the close phylogenetic relationship of the six $P y C D H$ paralogs, especially the high sequence similarities in the two paralogous groups (PyCDH2 and PyCDH4, PyCDH5 and PyCDH6), we deduced that the most plausible explanation was that multiple gene duplication events leading to the acquirement of new $\mathrm{CDH}$ genes happened in the evolution of the P. yezoensis genome, accounting for the rapid accumulation of betaine for cell protection when encountering osmotic stresses.

Another prominent observation in P. yezoensis GB biosynthesis was that $\mathrm{CDH}$ paralogs exhibited simultaneous up-regulation at the onset of osmotic stress, and then dropped to the basal level when dehydration was ongoing. Three of them also showed transcriptional elevation in re-hydration. The coordinated transcriptional pattern suggested the existence of an elaborate regulatory mechanism that could be induced by osmotic stress. In addition to this, our analyses showed an increase in the enzyme activity for $\mathrm{CDH}$ under moderate and severe osmotic stress in P. yezoensis. Further study of the cis-regulatory elements or conserved DNA motifs located in their promoter regions would help to illustrate the key components and their regulatory network in response to osmotic stresses.

\section{Materials and Methods}

\subsection{Algal Material and Stress Treatment}

The pure line RZ58 of P. yezoensis, established by clonal cultivation of an isolated single somatic cell and self-fertilization in the laboratory, was used for the experiments. Fresh leafy gametophytes were cultured in bubbling natural seawater with Provasoli's enrichment solution medium (PES) under $50 \mu \mathrm{mol}$ photons $\mathrm{m}-2 \mathrm{~s}-1$ at $10 \pm 1{ }^{\circ} \mathrm{C}$ and a 12:12 light (dark photoperiod before use). The seawater was bubbled continuously with filter-sterilized air and renewed every three days. 
P. yezoensis blades were subjected to dehydration by exposing them to the air for a period of time. They were collected when water loss was weighed to be $30 \pm 5 \%, 50 \pm 5 \%$, and $80 \pm 5 \%$, respectively. For rehydration, severely dehydrated samples (water loss at $80 \pm 5 \%$ ) were transferred back into seawater, and collected $0.5 \mathrm{~h}$ later. Water loss was determined according to the method by Kim et al. [28]. Three biological replicates were produced for treatment. After harvesting and weighing, the samples were immediately frozen in liquid nitrogen and stored individually at $-80^{\circ} \mathrm{C}$ until use.

\subsection{RNA Isolation and cDNA Synthesis}

Total RNA was extracted from the thallus using the Plant RNA Kit (Omega, U.S.) in accordance with the manufacturer's instructions. RNA degradation and contamination were monitored on $1 \%$ agarose gels. Purity was evaluated using a NanoPhotometer spectrophotometer (IMPLEN, CA, U.S.). RNA concentration was measured using a Qubit RNA Assay Kit and Qubit 2.0 Fluorometer (Life Technologies, CA, U.S.). RNA integrity was assessed using an RNA Nano 6000 Assay Kit and the Bioanalyzer 2100 system (Agilent Technologies, CA, U.S.). The cDNA templates were synthesized using a First Strand cDNA Synthesis Kit (Roche, Germany) according to the manufacturer's instructions. Subsequently, $1 \mu \mathrm{g}$ total RNA was used to synthesize the first-strand cDNA. The resulting cDNA mixture was diluted 10 times by adding nuclease-free water and stored at $-20^{\circ} \mathrm{C}$.

\subsection{Quantitative Real-Time PCR (RT-PCR)}

Sequence-specific primers of PyBADH, PyPEAMT, and PyCDHs, based on their multiple alignments, were designed using the Primer 5.0 software (Premier Biosoft International, USA) (Table 2). Quantitative real-time PCR (RT-qPCR) was performed with Light Cycler480 (Roche, Germany). $P y U B C$ was simultaneously used as internal control to normalize the amount of messenger RNA (mRNA) in each reaction. The cycling parameters were as follows: $95^{\circ} \mathrm{C}$ for $5 \mathrm{~min}$, followed by 45 cycles of $95^{\circ} \mathrm{C}$ for $10 \mathrm{~s}, 60^{\circ} \mathrm{C}$ for $10 \mathrm{~s}$, and $72{ }^{\circ} \mathrm{C}$ for $20 \mathrm{~s}$. The mean amplification efficiency of each primer pair was checked with the LightCycle ${ }^{\circledR} 480$ gene scanning software version 1.5 (Roche, Germany). The $2-\Delta \Delta \mathrm{Ct}$ method was used to assess the expression of relative genes [29]. The qPCR results shown are the average $( \pm \mathrm{SD})$ of three biological repeats.

Table 2. Information of primers.

\begin{tabular}{|c|c|c|}
\hline Primer Name & GenBank Entry & Sequence Information $\left(5^{\prime}-3^{\prime}\right)$ \\
\hline BADH & MK294535 & $\begin{array}{l}\text { F: GCGTCCCTGCGAGCCACTCAC } \\
\text { R: CCGTGTCAAAGGGGATAACCGT }\end{array}$ \\
\hline PEAMT & MK294536 & $\begin{array}{l}\text { F: CTCTTCGCACCCGTGACCTG } \\
\text { R: TGTCCAGGTAGGCGTCCGAG }\end{array}$ \\
\hline $\mathrm{CDH}-1$ & MK294537 & $\begin{array}{c}\text { F: GAACCGTTTTCGCCCTATCGC } \\
\text { R: CGCCACGCCCTTGACCC }\end{array}$ \\
\hline $\mathrm{CDH}-2$ & MK294538 & $\begin{array}{c}\text { F: CCGCATTGTCTGGGCTGCA } \\
\text { R: GACGCATCAACCACCCACAAGT }\end{array}$ \\
\hline $\mathrm{CDH}-3$ & MK294539 & $\begin{array}{l}\text { F: GCGGTGGGCACCTGCCGGAT } \\
\text { R: GGCGTTGGTGTTGCCACTCC }\end{array}$ \\
\hline $\mathrm{CDH}-4$ & MK294540 & $\begin{array}{l}\text { F: CCGAGTGACCACAGGCGA } \\
\text { R: AGCAGGTTGGTCTCCACACG }\end{array}$ \\
\hline UBC & ACI47322.1 & $\begin{array}{l}\text { F: TCACAACGAGGATTTACCACC } \\
\text { R: GAGGAGCACCTTGGAAACG }\end{array}$ \\
\hline
\end{tabular}

\subsection{GB Extraction and Liquid Chromatography Analysis}

About $200 \mathrm{mg}$ of the material was ground with liquid nitrogen, then pre-cooled with $1 \mathrm{~mL}$ methanol, and ultrasonically extracted for $30 \mathrm{~min}$. This was centrifuged for $10 \mathrm{~min}$ at $10,000 \times \mathrm{g}$ 
(HITACHI, Tokyo, Japan) and the supernatant was mixed after repeating twice, concentrated to dry under a nitrogen blower (NDK200-2, Miulab, Hangzhou, China), dissolved by vortex oscillation in $1 \mathrm{~mL}$ water was added, and then filtered with $0.22-\mu \mathrm{m}$-pore filter membranes (Millipore).

\subsection{Detecting Content of $G B$}

The content of betaine was determined by HPLC (L-3000, RIGOL, Beijing, China). By comparing the peak area of the samples with that of the standards (Shanghai yuanye, Bio-Technology, Shanghai, China), the content of the samples was calculated.

\subsection{Determination of Choline Dehydrogenase Activity}

The choline dehydrogenase activity was detected using a Plant CHDH ELISA kit (Mlbio, Shanghai, China). Phosphate buffer saline (PBS) $(100 \mu \mathrm{g}$ material, $900 \mu \mathrm{L}$ PBS) was added into a tube after grinding samples with liquid nitrogen. The samples were mixed and then centrifuged for $20 \mathrm{~min}$ (Hitachi, Tokyo, Japan) at $2500 \times g$ and the supernatant was taken. The kit was removed from the $4{ }^{\circ} \mathrm{C}$ environment and balanced at room temperature for $25 \mathrm{~min}$. The standard wells, sample wells, and blank wells were set, and the absorbance (OD) of each well was measured at $450 \mathrm{~nm}$ by zero-setting the blank well.

\subsection{Statistics}

Data were analyzed using the Origin Pro 9.0 software (Origin Lab, Northampton, MA, USA). Datasets were normalized to test the statistical significance of the treatment effect and a one-way ANOVA was performed using the SPSS 22.0 software (IBM, USA). Significant differences between group means were determined as $p<0.05$ via Tukey's and Fisher's tests.

\section{Conclusions}

In this study, we reconstructed the biosynthesis pathway of glycine betaine in the economically important seaweed P. yezoensis and experimentally detected the existence and rapid accumulation of GB as well as the transcriptional regulation under desiccation stress. The extensive illustration of the genetic foundation in the GB biosynthesis in P. yezoensis will undoubtedly further our understanding of the adaptive strategies of intertidal seaweeds in their evolutionary history and provide precious genetic resources for genetic engineering of algae and plants for abiotic stress tolerance.

Author Contributions: Data curation, N.C.; Funding acquisition, Y.M.; Methodology, N.C., R.C., and X.G.; Project administration, Y.M.; Resources, D.W.; Supervision, D.W. and M.C.; Writing-original draft, N.C. and D.W.; Writing-review \& editing, D.W. and Y.M.

Funding: This work was financially supported by the National Key R\&D Program of China (2018YFD0900106, 2018YFC1406700), National Natural Science Foundation of China (Grant No. 31401116, 31672641), the China Postdoctoral Science Foundation Grant (2016M602191), the Marine S\&T Fund of Shandong Province for Pilot National Laboratory for Marine Science and Technology (Qingdao) (No. 2018SDKJ0302-5), the Fundamental Research Funds for the Central Universities (201762016), the Program for Chinese Outstanding Talents in Agriculture Scientific Research, and the Project of National Infrastructure of Fishery Germplasm Resources (2018DKA30470). These funding bodies had no role in the study design, analysis, decision to publish, or preparation of the manuscript.

Conflicts of Interest: The authors declare no conflict of interest.

\section{References}

1. Abraham, B. Effective use of water (EUW) and not water-use efficiency (WUE) is the target of crop yield improvement under drought stress. Field Crops Res. 2009, 112, 119-123. [CrossRef]

2. Chaves, M.M.; Maroco, J.P.; Pereira, J.S. Understanding plant responses to drought - from genes to the whole plant. Funct. Plant Biol. 2003, 30, 239-264. [CrossRef] 
3. Reynolds, M.; Foulkes, J.; Furbank, R.; Griffiths, S.; King, J.; Murchie, E.; Parry, M.; Slafer, G. Achieving yield gains in wheat. Plant Cell Environ. 2012, 35, 1799-1823. [CrossRef] [PubMed]

4. Lu, G.H.; Ren, D.L.; Wang, X.Q.; Wu, J.K.; Zhao, M.S. Evaluation on drought tolerance of maize hybrids in China. J. Maize Sci. 2010, 3, 20-24. [CrossRef]

5. Ashraf, M. Review Inducing drought tolerance in plants: Recent advances. Biotechnol. Adv. 2010, 28, $169-183$. [CrossRef] [PubMed]

6. Ahmad, R.; Kim, M.D.; Back, K.H.; Kim, H.S.; Lee, H.S.; Kwon, S.Y.; Murata, N.; Chung, W.I.; Kwak, S.S. Stress-induced expression of choline oxidase in potato plant chloroplasts confers enhanced tolerance to oxidative, salt, and drought stresses. Plant Cell Rep. 2008, 27, 687-698. [CrossRef] [PubMed]

7. Chen, T.H.; Murata, N. Enhancement of tolerance to abiotic stress by metabolic engineering of betaines and other compatible solutes. Curr. Opin. Plant Biol. 2002, 5, 250-257. [CrossRef]

8. Takabe, T.; Rai, V.; Hibino, T. Metabolic engineering of glycinebetaine. In Abiotic Stress Tolerance in Plants: Toward the Improvement of Global Environment and Food; Springer: Dordrecht, The Netherlands, 2006; pp. 137-151.

9. Chen, T.H.; Murata, N. Glycinebetaine: An effective protectant against abiotic stress in plants. Trends Plant Sci. 2008, 13, 499-505. [CrossRef]

10. Yang, X.; Liang, Z.; Lu, C. Genetic engineering of the biosynthesis of glycine betaine enhances photosynthesis against high temperature stress in transgenic tobacco plants. Plant Physiol. 2005, 138, 2299-2309. [CrossRef]

11. Yang, X.; Wen, X.; Gong, H.; Lu, Q.; Yang, Z.; Tang, Y.; Liang, Z.; Lu, C. Genetic engineering of the biosynthesis of glycinebetaine enhances thermo tolerance of photosystem II in tobacco plants. Planta 2007, 225, 719-733. [CrossRef]

12. Carillo, P.; Mastrolonardo, G.; Nacca, F.; Parisi, D.; Verlotta, A.; Fuggi, A. Nitrogen metabolism in durum wheat under salinity: Accumulation of proline and glycine betaine. Funct. Plant Biol. 2008, 35, 412-426. [CrossRef]

13. Kewei, Z.; Juan, W.; Lijun, L.; Wenju, F.; Ning, G.; Sulian, L. Increased Chilling Tolerance Following Transfer of a betA Gene Enhancing Glycinebetaine Synthesis in Cotton (Gossypium hirsutum L.). Plant Mol. Biol. Rep. 2012, 30, 1158-1171. [CrossRef]

14. Rontein, D.; Basset, G.; Hanson, A.D. Metabolic engineering of osmoprotectant accumulation in plants. Metab. Eng. 2002, 4, 49-56. [CrossRef] [PubMed]

15. Chen, T.H.; Murata, N. Glycinebetaine protects plants against abiotic stress: Mechanisms and biotechnological applications. Plant Cell Environ. 2015, 34, 1-20. [CrossRef] [PubMed]

16. Rathinasabapathi, B.; Burnet, M.; Russell, B.L.; Gage, D.A.; Liao, P.O.; Nye, G.J.; Scott, P.; Golbeck, J.H.; Hanson, A.D. Choline monooxygenase, an unusual iron-sulfur enzyme catalyzing the first step of glycine betaine synthesis in plants: Prosthetic group characterization and cDNA cloning. Proc. Natl. Acad. Sci. USA 1997, 94, 3454-3458. [CrossRef] [PubMed]

17. Burnet, M.; Lafontaine, P.J.; Hanson, A.D. Assay, purification, and partial characterization of choline monooxygenase from spinach. Plant Physiol. 1995, 108, 581-588. [CrossRef]

18. Salvi, F.; Gadda, G. Human choline dehydrogenase: Medical promises and biochemical challenges. Arch. Biochem. Biophys. 2013, 537, 243-252. [CrossRef]

19. Kageyama, H.; Tanaka, Y.; Takabe, T.T. Biosynthetic pathways of glycinebetaine in Thalassiosira pseudonana; functional characterization of enzyme catalyzing three-step methylation of glycine. Plant Physiol. Biochem. 2018, 127, 248-255. [CrossRef]

20. McNeil, S.D.; Nuccio, M.L.; Ziemak, M.J.; Hanson, A.D. Enhanced synthesis of choline and glycine betaine in transgenic tobacco plants that overexpress phosphoethanolamine N-methyltransferase. Proc. Natl. Acad. Sci. USA 2001, 98, 10001-10005. [CrossRef]

21. Lorenzin, D.; Webb, C.; Summers, P.S.; Weretilnyk, E.A. Enzymes of choline synthesis in diverse plants: Variation in phosphobase N-methyltransferase activities. Can. J. Bot. 2001, 79, 897-904. [CrossRef]

22. Waditee, R.; Bhuiyan, M.N.H.; Rai, V.; Aoki, K.; Tanaka, Y.; Hibino, T.; Suzuki, S.; Takano, J.; Jagendorf, A.T.; Takabe, T.; et al. Genes for direct methylation of glycine provide high levels of glycine betaine and abiotic-stress tolerance in Synechococcus and Arabidopsis. Proc. Natl. Acad. Sci. USA 2005, 102, 1318-1323. [CrossRef] [PubMed] 
23. Waditee-Sirisattha, R.; Singh, M.; Kageyama, H.; Sittipol, D.; Rai, A.K.; Takabe, T. Anabaena sp. PCC7120 transformed with glycine methylation genes from Aphanothece halophytica synthesized glycine betaine showing tolerance to salt. Arch. Microbiol. 2012, 194, 909-914. [CrossRef] [PubMed]

24. Sutherland, J.E.; Lindstrom, S.C.; Nelson, W.A.; Brodie, J.; Lynch, M.D.J.; Hwang, M.S.; Choi, H.; Miyata, M.; Kikuchi, N.; Oliveira, M.C.; et al. A new look at an ancient order: Generic revision of the Bangiales (Rhodophyta). J. Phycol. 2011, 47, 1131-1151. [CrossRef] [PubMed]

25. Xu, Y.; Chen, C.; Ji, D.; Hang, N.; Xie, C. Proteomic profile analysis of Pyropia haitanensis in response to high-temperature stress. J. Appl. Phycol. 2014, 26, 607-618. [CrossRef]

26. Blouin, N.A.; Brodie, J.A.; Grossman, A.C.; Xu, P.; Brawley, S.H. Porphyra: A marine crop shaped by stress. Trends Plant Sci. 2011, 16, 29-37. [CrossRef] [PubMed]

27. Weretilnyk, E.A.; Hanson, A.D. Molecular cloning of a plant betaine-aldehyde dehydrogenase, an enzyme implicated in adaption to salinity and drought. Proc. Natl. Acad. Sci. USA 1990, 87, 2745-2749. [CrossRef] [PubMed]

28. Kim, J.K.; Kraemer, G.P.; Yarish, C. Comparison of growth and nitrate uptake by New England Porphyra species from different tidal elevations in relation to desiccation. Phycol. Res. 2009, 57, 152-157. [CrossRef]

29. Livak, K.J.; Schmittgen, T.D. Analysis of relative gene expression data using real-time quantitative PCR and the 22DDCT method. Methods 2001, 25, 402-408. [CrossRef]

(C) 2019 by the authors. Licensee MDPI, Basel, Switzerland. This article is an open access article distributed under the terms and conditions of the Creative Commons Attribution (CC BY) license (http://creativecommons.org/licenses/by/4.0/). 